\title{
Dissipation in a finite-size bath
}

\author{
A. Carcaterra ${ }^{1, *}$ and A. Akay ${ }^{2, \dagger}$ \\ ${ }^{1}$ Department of Mechanical and Aerospace Engineering, University of Rome "La Sapienza," I-00184 Rome, Italy \\ ${ }^{2}$ Department of Mechanical Engineering, Bilkent University, 06800 Bilkent, Ankara, Turkey
}

(Received 10 March 2011; published 18 July 2011)

\begin{abstract}
We investigate the interaction of a particle with a finite-size bath represented by a set of independent linear oscillators with frequencies that fall within a finite bandwidth. We discover that when the oscillators have particular frequency distributions, the finite-size bath behaves much as an infinite-size bath exhibiting dissipation properties and thus allowing irreversible energy absorption from a particle immersed in it. We also present a reinterpretation of the Langevin equation using a perturbation approach in which the small parameter represents the inverse of the number of oscillators in the bath, elucidating the relationship between finite-size and infinite-size bath responses.
\end{abstract}

DOI: 10.1103/PhysRevE.84.011121

PACS number(s): 05.40.Jc, 05.10.Gg, 05.70.Ln

\section{INTRODUCTION}

In its most fundamental form, classical or quantum dissipation can be considered as an interaction of a particle with its environment. In their seminal work, Feynman and Vernon [1] formulated such interactions using the influence functional method that was later adapted by Caldeira and Leggett $[2,3]$ to the study of Brownian motion. In these and other studies that followed, the environment is modeled as a thermal bath consisting of a continuous set of noninteracting, linear independent oscillators into which energy flows from the particle of interest [4]. These formulations also yield the fluctuation-dissipation theorem and provide details for the phenomenological parameters that appear in the descriptions of Brownian motion [5] and the Langevin equation, but only for the limiting case of an infinite-size heat bath. The presumption of an infinite number of linear oscillators in the heat bath permits irreversible energy flow into the bath, which then acts as an energy sink. In cases where the bath size is small, such as those surrounding nanoscale devices, the presence of an infinite number or a continuous distribution of linear oscillators may not be justified [6]. In this paper we report frequency distributions for the oscillators that make a finite-size bath behave very much like an infinite-size bath. Through these frequency distributions it becomes possible to model dissipation and irreversible energy absorption from a particle in a finite-size bath with a set of independent linear oscillators. These developments also lead to a reinterpretation of the Langevin equation, presented here using a perturbation approach that elucidates the relationship between finite-size and infinite-size bath responses.

\section{FINITE BATH}

Following Refs. [6,7], the generalized Langevin equation for a particle of mass $M$ and angular frequency $\Omega$, with

\footnotetext{
*a.carcaterra@dma.ing.uniroma1.it

${ }^{\dagger}$ akay@bilkent.edu.tr; Also at: Department of Mechanical Engineering, Carnegie Mellon University, Pittsburgh, Pennsylvania 15213, USA.
}

the partitioned force by the oscillators in the bath, has the form

$$
M \ddot{q}(t)+M \Omega^{2} q(t)+\int_{0}^{t} \Gamma(\tau) \dot{q}(t-\tau) d \tau=\Pi(t),
$$

where $q(t)$ describes the test particle motion with quiescent initial conditions $q(0)=0$ and $\dot{q}(0)=0$ and the average of the fluctuating force is $\langle\Pi(t)\rangle=0$, where \langle\rangle represents the statistical average over the ensemble. The dissipative term $\Gamma(\tau)$ represents the correlation function of the bath and can also be considered as its impulse response. For the case of a finite-size bath that consists of linear noninteracting oscillators, each described by its mass $m$ and angular frequency $\omega_{i}, \Gamma(\tau)$ has the form

$$
\Gamma(\tau)=\sum_{i=1}^{N} m \omega_{i}^{2} \cos \omega_{i} \tau,
$$

where $m=m_{B} / N$ and $m_{B}$ and $N$ represent the total mass and number of particles in the thermal bath, respectively. Thus

$$
\Gamma(\tau)=\frac{1}{N} \sum_{i=1}^{N} G\left(\omega_{i}\right) \cos \omega_{i} \tau=\frac{1}{N} \sum_{i=1}^{N} \varphi_{i}(\tau),
$$

where $G\left(\omega_{i}\right)=m_{B} \omega_{i}^{2}$. As discussed above, under conditions when a continuous distribution is justified for the bath oscillators, the summation in the correlation function is replaced by an integral as

$$
\Gamma_{\infty}(\tau)=\lim _{N \rightarrow \infty} \Gamma(\tau)=\int_{\omega_{\min }}^{\omega_{\max }} \frac{d m}{d \omega} \omega^{2} \cos \omega \tau d \omega,
$$

which now represents the combined response of an infinite number of resonators, each with a vanishingly small mass and a frequency in the bandwidth $\left[\omega_{\min }, \omega_{\max }\right]$, where $d m / d \omega$ represents the spectral mass density of the bath. Replacing the summation by an integral effectively increases the number of oscillators to infinity, affording the heat bath a dissipative character.

In general, however, harmonic series such as $\Gamma(\tau)$ have properties similar to those of almost-periodic functions and exhibit recurrence [8], precluding use of independent linear oscillators to model dissipation in a finite-size heat bath, except for early times of observation for $\Gamma(\tau)$ [9]. In this paper we 
report a method that yields a class of frequency distributions that enable a finite set of independent oscillators to mimic very closely the dissipation characteristics of an infinite-size bath, reducing and nearly eliminating the effects of recurrence. We use a probabilistic approach, based on the Cramér-Rao minimum variance bound (MVB) theorem [10,11] and the Pitman-Koopman theorem [12,13], to minimize the distance between the summation and the corresponding integral representation of the correlation.

We assume the frequencies $\omega_{i}$ in Eq. (2) as samples of the random variable $\omega$ with a probability density function $p_{\omega}(\omega)$. Then $\Gamma(\tau)$ in Eq. (3) can be considered as the estimator, obtained through the arithmetic average of the samples $\varphi_{i}(\tau)=$ $G\left(\omega_{i}\right) \cos \left(\omega_{i} \tau\right)$ for the expected value $\bar{\varphi}(\tau)$ of the random variable $\varphi(\tau)=G(\omega) \cos (\omega \tau)$ between the lower and upper frequency limits $\omega_{\min }=\omega_{1}$ and $\omega_{\max }=\omega_{N}$. In terms of the probability density function (PDF) $p_{\varphi}(\varphi)$, the expected value of $\varphi$ can be expressed as

$$
\begin{aligned}
\bar{\varphi}(\tau) & =\int_{-G_{\max }}^{G_{\max }} p_{\varphi}(\varphi) \varphi d \varphi \\
& =\int_{\omega_{\max }}^{\omega_{\max }} p_{\omega}(\omega) G(\omega) \cos \omega \tau d \omega=\bar{\Gamma}(\tau)
\end{aligned}
$$

since for unbiased estimators the mean of the estimator and the expected value are equal.

Integrating Eq. (5) by parts, it can be shown by an asymptotic expansion of the integral that $\bar{\Gamma}$ vanishes as $1 / \tau$, indicating dissipation:

$$
\lim _{\tau \rightarrow \infty} \bar{\Gamma}(\tau)=\lim _{\tau \rightarrow \infty}\left[\frac{1}{\tau}\left[p_{\omega}(\omega) G(\omega) \sin \omega \tau\right]_{\omega_{\min }}^{\omega_{\max }}+\mathcal{O}\left(\frac{1}{\tau}\right)\right]=0 .
$$

$\bar{\Gamma}$ decays with a double-frequency modulation, associated with the frequency bounds of the interval, which for a narrow bandwidth displays a decaying beat.

Unlike the average correlation $\bar{\Gamma}(\tau)$, which vanishes for long times, the correlation function $\Gamma(\tau)$ of the same bath is an almost periodic function and, in general, exhibits recurrence. For example, the case of a constant frequency gap $\Delta \omega=$ $\left(\omega_{\max }-\omega_{\min }\right) / N$, with $\omega_{i}=i \Delta \omega$, makes $\Gamma(\tau)$ periodic, with a period $2 \pi N /\left(\omega_{\max }-\omega_{\min }\right)$. The divergence between $\Gamma(\tau)$ and $\bar{\Gamma}(\tau)$ becomes more pronounced for small values of $N$. For the extreme case of $N=1, \Gamma(\tau)$ is a pure harmonic function, whereas $\bar{\Gamma}(\tau)$ still vanishes asymptotically. From a comparison of Eqs. (4) and (5) it can be seen that $\Gamma_{\infty}(\tau)$ approaches $\bar{\Gamma}(\tau)$ when the selected PDF of the frequencies of the bath is proportional to the mass spectral density, $d m / d \omega=p_{\omega} m_{B}$.

The estimator $\Gamma(\tau)$ and the expected value $\bar{\Gamma}(\tau)$ display the differences between discrete and continuous systems as expressed by the summation and integral, respectively. We show that frequencies for $\varphi_{i}$ can be selected such that the time response of the estimator $\Gamma(\tau)$ follows the expectation $\bar{\Gamma}(\tau)$ as closely as possible. This selection is accomplished using the minimum variance bound theorem, which relates the estimator $\Gamma(\tau)$ for the mean of the random variable $\varphi$ to its expected value through probability density functions $p_{\varphi}[10-13]$. In fact, the smaller the variance $\operatorname{var}[\Gamma(\tau)]$ is, the closer the estimator $\Gamma(\tau)$ approaches $\bar{\Gamma}(\tau)$. However, there is a limit to how close they can become, as established by a lowest bound given by the Cramér-Rao inequality. Furthermore, there exist particular populations of heat baths, described by their probability density functions $p_{\varphi}$ or $p_{\omega}$, that minimize $\operatorname{var}[\Gamma(\tau)]$ at $\tau=0$ where the correlation is maximum, and thus can exactly match this lowest bound [12,13]. For these populations, the estimator $\Gamma(0)$ approaches its expected value $\bar{\Gamma}(0)$ as closely as possible, making the finite-size bath emulate the properties of an infinite-size bath in the closest possible manner.

Using the simplified notations $\varphi_{i}=\varphi_{i}(0)$ and $\bar{\Gamma}=\bar{\Gamma}(0)$ for brevity, where $\varphi_{i}$ are independent observed samples of $\varphi$ evaluated at $\tau=0$, from the estimation theory the likelihood function $L$ for the entire system is introduced as

$$
L\left(\varphi_{1}, \varphi_{2}, \ldots, \varphi_{N} \mid \bar{\Gamma}\right)=p_{\varphi}\left(\varphi_{1} \mid \bar{\Gamma}\right), p_{\varphi}\left(\varphi_{2} \mid \bar{\Gamma}\right) \ldots p_{\varphi}\left(\varphi_{N} \mid \bar{\Gamma}\right)
$$

and $L$ satisfies the conditions

$$
\begin{gathered}
\int_{-G_{\max }}^{G_{\max }} L\left(\varphi_{1}, \ldots, \varphi_{N} \mid \bar{\Gamma}\right) d \varphi_{1}, \ldots, d \varphi_{N}=1, \\
\int_{-G_{\max }}^{G_{\max }} L\left(\varphi_{1}, \ldots, \varphi_{N} \mid \bar{\Gamma}\right) \Gamma\left(\varphi_{1}, \ldots, \varphi_{N}\right) d \varphi_{1}, \ldots, d \varphi_{N}=\bar{\Gamma} .
\end{gathered}
$$

Combining the conditions in Eq. (8) yields

$$
\int_{-G_{\max }}^{G_{\max }} \frac{\partial L}{\partial \bar{\Gamma}}(\Gamma-\bar{\Gamma}) d \varphi_{1}, \ldots, d \varphi_{N}=1 .
$$

Equation (9), together with the Schwartz inequality, produces a lower bound for the variance of $\Gamma$ known as the Cramér-Rao minimum variance bound:

$$
\begin{aligned}
\operatorname{var}(\Gamma) & =\int_{-G_{\max }}^{G_{\max }} L(\Gamma-\bar{\Gamma})^{2} d \varphi_{1}, \ldots, d \varphi_{N} \\
& \geqslant \frac{1}{\int_{-G_{\max }}^{G_{\max }}(1 / L)(\partial L / \partial \bar{\Gamma})^{2} d \varphi_{1}, \ldots, d \varphi_{N}},
\end{aligned}
$$

where the denominator is the Fisher information functional. The class of probability density functions that exactly match the lowest bound of this inequality is obtained from Eq. (9) by noting that the two functions $(1 / \sqrt{L})(\partial L / \partial \bar{\Gamma})$ and $\sqrt{L}(\Gamma-\bar{\Gamma})$ must be proportional:

$$
\frac{1}{L} \frac{\partial L}{\partial \bar{\Gamma}}=a(\bar{\Gamma})(\Gamma-\bar{\Gamma}),
$$

where the proportionality constant $a$, in the most general case, depends on the expectation $\bar{\Gamma}$ but is independent of the integration variable $\varphi_{i}$. Using the Pittman-Koopman theorem $[12,13]$, the general form of the function $p_{\varphi}$ is found as

$$
p_{\varphi}(\varphi \mid \bar{\Gamma})=h(\varphi) \exp [\alpha(\bar{\Gamma}) \varphi-\beta(\bar{\Gamma})],
$$

which depends on the two arbitrary functions $h(\varphi)$ and $a(\bar{\Gamma})$, through $\alpha(\bar{\Gamma})=(1 / N) \int a(\bar{\Gamma}) d \bar{\Gamma}$ and $\beta(\bar{\Gamma})=$ $(1 / N) \int a(\bar{\Gamma}) \bar{\Gamma} d \bar{\Gamma}$, subject only to the constraints in Eq. (8).

Equation (12), which represents the so-called canonical exponential family, embodies a large class of functions that exactly match the lower bound on the right-hand side of Eq. (10), producing an equality. Because the changes in the selected function $p_{\varphi}$, and thus $L(\varphi \mid \bar{\Gamma})$, also change the minimum bound value on the right-hand side of Eq. (10), in 
order to determine the distribution that produces the lowest among those lower bounds that satisfy the MVB theorem, we introduce the additional requirement that the change of variance with respect to $\bar{\Gamma}$ vanishes:

$$
\frac{\partial}{\partial \bar{\Gamma}}(\operatorname{var} \bar{\Gamma})=\frac{\partial}{\partial \bar{\Gamma}} \int_{-\mathrm{G}_{\max }}^{\mathrm{G}_{\max }} \mathrm{L}(\Gamma-\bar{\Gamma})^{2} \mathrm{~d} \varphi_{1}, \ldots, \mathrm{d} \varphi_{\mathrm{N}}=0
$$

A variational approach using Lagrange multipliers, subject to the constraints in Eq. (8), produces

$$
\frac{d a}{d \bar{\Gamma}}=0 .
$$

Thus the additional requirement in Eq. (13) forces the coefficient $a$ in Eq. (11) to be constant. Then the distribution given by Eq. (12) takes the form of the special case of a natural exponential family:

$$
p_{\varphi}(\varphi \mid \bar{\Gamma})=\Psi(\varphi) \exp \left(-\frac{a}{2 N}(\varphi-\bar{\Gamma})^{2}\right),
$$

where $\Psi(\varphi)=h(\varphi) \exp \left(a \varphi^{2} / 2 N\right)$.

If in the interval $\left[\omega_{\min }, \omega_{\max }\right]$ the function $\varphi(0)=G(\omega)$ varies with frequency monotonically, as in the case of linear noninteracting resonators, it holds that

$$
p_{\varphi}(\varphi \mid \bar{\Gamma})\left|\frac{d \varphi}{d \omega}\right|=p_{\omega}(\omega \mid \bar{\Gamma}) .
$$

The probability density function $p_{\omega}(\omega)$ matching the lowest bound of the MVB theorem can be found as

$$
p_{\omega}(\omega \mid \bar{\Gamma})=\Psi(\omega)\left|\frac{d G(\omega)}{d \omega}\right| \exp \operatorname{del}\left\{\left(-\frac{a}{2 N}[G(\omega)-\bar{\Gamma}]^{2}\right\}\right) \text {. }
$$

For a heat bath of linear noninteracting oscillators for which $G=m_{B} \omega^{2}$,

$$
p_{\omega}(\omega \mid \bar{\Gamma})=\Psi(\omega) 2 m_{B} \omega \exp \left(-\frac{a}{2 N}\left(m_{B} \omega^{2}-\bar{\Gamma}\right)^{2}\right) .
$$

\section{LANGEVIN EQUATION FOR A FINITE BATH}

The distribution in Eq. (18) is born from a comparison of $\Gamma$ and $\bar{\Gamma}$ considering only the dissipative role of a finite-size heat bath and how it can be modeled by a finite number of independent linear oscillators. An analogous development for the fluctuation term $\Pi(t)$ in Eq. (1) helps recast the Langevin equation for finite-size baths:

$$
\Pi(t)=\sum_{i=1}^{N} m \omega_{i}^{2}\left(x_{i 0} \cos \omega_{i} t+\frac{\dot{x}_{i 0}}{\omega_{i}} \sin \omega_{i} t\right),
$$

where $x_{i 0}$ and $\dot{x}_{i 0}$ represent the initial conditions of the heat bath and it is assumed that $q(0)=\dot{q}(0)=0$.

Rather than treating only the dissipative part of the environment (bath) or using a contracted definition for it as is generally done, dissipation, fluctuation, and memory effects can be directly obtained for large $N$ values by a formal solution of the stochastic equations as shown in Ref. [5]. We present an alternative approach that relates dissipation and fluctuation and avoids the need to replace $\Gamma$ with $\bar{\Gamma}$ by invoking the large- $N$ argument. By introducing a perturbation parameter $\epsilon=1 / N$, the dissipative and random parts of the bath can be expressed through an expansion:

$$
\begin{gathered}
\Gamma(t)=\bar{\Gamma}(t)+\epsilon \gamma_{1}(t)+\epsilon^{2} \gamma_{2}(t)+\cdots, \\
\Pi(t)=\bar{\Pi}(t)+\epsilon \pi_{1}(t)+\epsilon^{2} \pi_{2}(t)+\cdots,
\end{gathered}
$$

where the respective expectations are

$$
\begin{gathered}
\bar{\Gamma}(t)=\int_{\omega_{\min }}^{\omega_{\max }} m_{B} p_{\omega}(\omega) \omega^{2} \cos \omega t d \omega, \\
\bar{\Pi}(t)=\int_{\omega_{\min }}^{\omega_{\max }} m_{B} p_{\omega}(\omega) \omega^{2} \cos \omega t \\
\times\left(x_{0}(\omega) \cos \omega+\frac{\dot{x}_{0}(\omega)}{\omega} \sin \omega t\right) d \omega .
\end{gathered}
$$

The corresponding motion for the test particle is similarly expressed as

$$
q(t)=q_{0}(t)+\epsilon q_{1}(t)+\epsilon^{2} q_{2}(t)+\cdots,
$$

where the perturbations represent the differences of a finitesize bath from the infinite bath. Substitution of these expressions into Eqs. (1) and (19) produces a set of equations similar in form to the Langevin equation:

$$
\begin{array}{r}
M \ddot{q}_{0}(t)+M \Omega^{2} q_{0}(t)+\int_{0}^{t} \bar{\Gamma}(\tau) \dot{q}_{0}(t-\tau) d \tau=\bar{\Pi}(t), \\
M \ddot{q}_{1}(t)+M \Omega^{2} q_{1}(t)+\int_{0}^{t} \bar{\Gamma}(\tau) \dot{q}_{1}(t-\tau) d \tau \\
=\pi_{1}(t)-\int_{0}^{t} \gamma_{1}(\tau) \dot{q}_{0}(t-\tau) d \tau,
\end{array}
$$

etc., for higher-order equations. For an infinite-size bath, $\epsilon=0$ and the set of Eqs. (22a) and (22b) reduce to the first in the set in which the correlation and the fluctuation functions both take their expected values. As a result of the evanescent behavior of $\bar{\Pi}(t)$, together with the asymptotic dissipation induced by $\bar{\Gamma}(t)$ in the first Eq. (22a), in the long times $q_{0}(t)$ vanishes. It follows that in Eq. (22b) the integral on the right-hand side also vanishes and $\pi_{1}(t)$ approaches $\Pi(t)$ as the dominant term in the expansion series leading to the first-order approximation of the motion of the test particle:

$$
M \ddot{q}(t)+M \Omega^{2} q(t)+\int_{0}^{t} \bar{\Gamma}(\tau) \dot{q}(t-\tau) d \tau=\Pi(t),
$$

which represents the generalized Langevin equation as does Eq. (1), except now the correlation is represented by $\bar{\Gamma}(\tau)$, the expectation rather than the estimator $\Gamma(\tau)$. The set of equations of Eq. (22) helps establish the relationship between dissipation and fluctuation for finite-size baths in the context of the classical Langevin equation.

\section{CONCLUDING REMARKS}

It is worth noting that the minimum variance bound requirement is, under some conditions, equivalent to the maximum entropy formulation as defined in information theory. It follows that the finite-size bath that behaves the closest to an infinite-size bath in accordance with the MVB 
criterion is the one for which $p_{\omega}$ represents a maximum entropy distribution. We can speculate that the distribution in Eq. (18) offers hints, and will be the basis, for further developments and links to other physical problems.

For example, the superposition of $N$ harmonic functions in the expression of $\Gamma(\tau)$ in Eq. (2) represents the impulse response of any linear system having natural frequencies $\omega_{i}$, such as a finite-length waveguide. The group velocity $c_{g}$ along the guide is proportional to the inverse of the state density $d N / d \omega$ and therefore is proportional to the inverse of $p_{\omega}$. Interpretation of the distribution in Eq. (18) for this case suggests that an asymptotically vanishing $\Gamma(\tau)$ can be obtained by special natural frequency distributions in the waveguide, physically corresponding to an almost vanishing group velocity in a given frequency bandwidth. Thus, by this interpretation, a wave stopping process is associated with Eq. (18) that makes the finite-length waveguide behave much as an infinite-length one. An extension of the results reported here can be applied to slowing down or stopping of waves in optics and acoustics and to other applications in which the Langevin approach is possible with a suitable selection of $G(\omega)$. We conjecture that the distribution in Eq. (17) can be used even for modeling relaxation in a harmonic glass disordered system such as glassy systems where the Langevin equation can be applied [14].

The findings of this paper also relate to some applications of Landau damping where a finite set of $N$ particles, with a given distribution of initial velocities, traveling along a harmonic force field, has an average kinetic energy similar to the expression in Eq. (2). Again, the law of large numbers is often invoked to replace the sum with an integral to produce Landau damping [15]. Similarly, the results of this paper are consistent with and applicable to the prediction of damping in complex structures [16].

Finally, in the family of functions described by Eq. (18) parametric studies of frequencies and through $\Psi(\omega), m, a$, and $\bar{\Gamma}$ and their relationship with the test particle frequency $\Omega$ and mass $M$ can lead to different types of particle-bath interactions. For example, the frequency $\Omega$ can be close to or far from the peak of the curve described by Eq. (18) or the total mass $m N$ of the bath can be larger than, comparable to, or smaller than the mass $M$ of the test particle. All these choices, while preserving the MVB theorem requirement, produce different energy interaction scenarios.
[1] R. P. Feynman and F. L. Vernon, Ann. Phys. (NY) 24, 118 (1963).

[2] A. O. Caldeira and A. J. Leggett, Physica A 121, 587 (1983).

[3] A. O. Caldeira and A. J. Leggett, Ann. Phys. (NY) 149, 374 (1983).

[4] G. W. Ford, M. Kac, and P. Mazur, J. Math. Phys. 6, 504 (1965).

[5] A. Einstein, Investigations on the Theory of the Brownian Movement (Dover, New York, 1956).

[6] Q. Wei, S. T. Smith, and R. Onofrio, Phys. Rev. E 79, 031128 (2009).

[7] M. Patriarca, Physica E 29, 243 (2005).

[8] H. Bohr, Almost-Periodic Functions (AMS Chelsea, Providence, RI, 1947).
[9] A. Carcaterra and A. Akay, J. Acoust. Soc. Am. 115, 683 (2004).

[10] H. Cramér, Mathematical Methods of Statistics, (Princeton University Press, Princeton, 1946).

[11] C. R. Rao, Bull. Calcutta Math. Soc. 37, 81 (1945).

[12] E. J. G. Pitman, Proc. Cambridge Philos. Soc. 32, 576 (1936).

[13] B. O. Koopman, Trans. Am. Math. Soc. 39, 399 (1936).

[14] G. Ruocco, F. Sette, R. Di Leonardo, G. Monaco, M. Sampoli, T. Scopigno, and G. Viliani, Phys. Rev. Lett. 84, 5788 (2000).

[15] S. H. Strogatz, R. E. Mirollo, and P. C. Matthews, Phys. Rev. Lett. 68, 2730 (1992).

[16] A. D. Pierce, V. W. Sparrow, and D. A. Russel, J. Vib. Acoust. 117, 339 (1995). 